\title{
Critical extinction exponents for a fast diffusion equation with nonlocal source and absorption
}

\section{Haixia Li and Yuzhu Han*}

\section{*Correspondence:}

hanyuzhu2003@163.com

Department of Mathematics, Jilin

University, Changchun, 130012, P.R.

China

\begin{abstract}
In this article, the authors apply the super-solution and sub-solution methods, instead of energy estimate methods, to investigate the critical extinction exponents for a fast diffusion equation with a nonlocal source and an absorption term. They give a classification of the exponents and coefficients for the solutions to vanish in finite time or not, which improve, in some sense, the results by Xu et al. (Bound. Value Probl. 2013:24 2013) and by Han et al. (Arch. Math. 97:353-363, 2011).
\end{abstract}

MSC: $35 \mathrm{~K} 55 ; 35 \mathrm{~K} 57$

Keywords: fast diffusion equation; critical exponents; extinction; super-solutions and sub-solutions

\section{Introduction}

In this paper, we investigate the following fast diffusion equation with a nonlocal source and an absorption term:

$$
\begin{cases}u_{t}=\Delta u^{m}+a \int_{\Omega} u^{q}(y, t) \mathrm{d} y-b u^{r}, & x \in \Omega, t>0, \\ u(x, t)=0, & x \in \partial \Omega, t>0, \\ u(x, 0)=u_{0}(x), & x \in \Omega,\end{cases}
$$

where $0<m<1, a, b, q, r>0, \Omega$ is a bounded domain in $R^{N}(N \geq 1)$ with smooth boundary $\partial \Omega$, and $u_{0} \in L^{\infty}(\Omega)$ is a nonnegative nontrivial function.

The equation in (1.1) is a fast diffusion equation perturbed by both a nonlocal source term and an absorption term, which describes the diffusion of concentration of some Newtonian fluids or the density of some biological species (see [1,2] and the references therein). What we are interested in here is the extinction in finite time of the nonnegative solutions $u(x, t)$ of (1.1), i.e. there exists a finite time $T>0$ such that the solution is nontrivial for $0<t<T$, but $u(x, t) \equiv 0$ for almost every $(x, t) \in \Omega \times[T, \infty)$. In this case, $T$ is called the extinction time. As one of the most important properties of solutions of evolutionary equations, extinction in finite time of solutions has been intensively studied by several authors (see [3-16] and the references therein). In particular, in a recent paper by $\mathrm{Xu}$ et al. [13], the authors investigated the extinction and non-extinction phenomena of solutions of Problem (1.1) and obtained the following result.

@2014 Li and Han; licensee Springer. This is an Open Access article distributed under the terms of the Creative Commons Attribution License (http://creativecommons.org/licenses/by/2.0), which permits unrestricted use, distribution, and reproduction in any medium, provided the original work is properly cited. 
Theorem 1.1 (Theorems 1-3 in [13]) Suppose $N \geq 1$. If $0<r<1$ and $0<m=q<1$, then the nonnegative nontrivial weak solution of Problem (1.1) vanishes in finite time for any nonnegative initial data provided that either $|\Omega|$ or a is sufficiently small; If $0<r<1$, then the nonnegative nontrivial weak solution of Problem (1.1) vanishes in finite time provided that $u_{0},|\Omega|$ or a is sufficiently small, and $q>C$ with $C$ being a positive constant depending only on $N$, $r$ and $m$; If $0<r<1$ and $m>q \geq r$, then the nonnegative nontrivial weak solution of Problem (1.1) vanishes in finite time for any nonnegative initial data provided that $b$ is sufficiently large.

It can be seen from the above theorem that the extinction of nonnegative nontrivial weak solutions to Problem (1.1) occurs when the absorption term is in some sense strong. However, when the absorption term is suitably weak, whether Problem (1.1) admits nonextinction solutions or not is not answered in [13]. On the other hand, it can be seen from [9] that $q=m$ is the critical extinction exponent when there is no absorption term. An interesting problem is whether the absorption term can change the critical extinction exponent. We know from a recent paper [17] by Liu et al. that the critical extinction exponent is not changed (at least when the source is local) when $r=1$. However, when the absorption term is nonlinear, i.e. when $r \neq 1$, the problem is open.

Motivated by the works mentioned above, we investigate the critical extinction exponents for Problem (1.1) by constructing suitable super and sub-solutions and give a more complete classification of exponents and coefficients for the solutions to vanish in finite time or not.

Let $\varphi(x)$ be the unique positive solution of the following linear elliptic problem:

$$
-\Delta \varphi(x)=1, \quad x \in \Omega ; \quad \varphi(x)=0, \quad x \in \partial \Omega .
$$

Throughout this paper, we denote

$$
M=\max _{x \in \bar{\Omega}} \varphi(x) \text { and } \quad \mu=\int_{\Omega} \varphi(x) \mathrm{d} x .
$$

By the strong maximum principle we know that $M, \mu>0$. Our main results are the following theorems.

Theorem 1.2 If $q>m$, then all the solutions $u(x, t)$ vanish in finite time for suitably small initial data $u_{0}(x)$. If $q=m$ and $a \mu<1$, then all the solutions $u(x, t)$ vanish in finite time for any nonnegative bounded initial data.

Theorem 1.3 If $r<\min \{q, 1\}$ or $r=q<1$ with $a|\Omega|<b$, then all the solutions $u(x, t)$ of Problem (1.1) vanish in finite time for appropriately small initial data $u_{0}(x)$.

Theorem 1.4 If $q<\min \{r, m\}$ or $r=q<m$ with $a \gamma>b$, then Problem (1.1) admits at least one non-extinction solution for any nonnegative initial data.

Here $\gamma>0$ will be given in the proof of Theorem 1.4.

Theorem 1.5 If $q=m<r$ with $a \mu>1$, then Problem (1.1) admits at least one nonextinction solution for any nonnegative initial data. 
Theorem 1.6 If $q=m<1 \leq r$ with $a \mu=1$, then (1.1) admits at least one non-extinction solution $u(x, t)$ for any strictly positive initial data $u_{0}(x)$. If $q=m<r<1$ with $a \mu=1$, then Problem (1.1) admits at least one extinction solution $u(x, t)$ for any nonnegative initial data. If $q=m<r<1$ and $a|\Omega| \leq \lambda_{1}$, then $u(x, t)$ vanishes in the sense that $\lim _{t \rightarrow+\infty}\|u(\cdot, t)\|_{m+1}=0$.

Remark 1.1 Comparing Theorems 1.2-1.6 with Theorem 1.1 we can see that our results complement those obtained in [13] since the case $r \geq 1$ is also considered in our paper. Moreover, according to Theorems 1.2, 1.5 and the first part of Theorem 1.6 it is easy to see that $q=m$ is the critical extinction exponent for Problem (1.1) when $r=1$, which is the same as the problems with local reaction terms (see [17]). However, when $r=1$ and $q=m$, for the nonlocal problem under consideration, the first eigenvalue $\lambda_{1}$ of $-\Delta$ in $\Omega$ no longer plays the same role as it does in the local case.

\section{Proof of the main results}

It is well known that the equation in (1.1) is degenerate if $m>1$ and singular if $0<m<1$, and therefore there is no classical solution in general. To state the definition of the weak solution, we first define the class of nonnegative testing functions

$$
\mathcal{F}=\left\{\xi: \xi \in C\left(\bar{Q}_{T}\right) \cap C^{2,1}\left(Q_{T}\right), \xi_{t}, \Delta \xi \in L^{2}\left(Q_{T}\right) ; \xi \geq 0,\left.\xi\right|_{\partial \Omega \times(0, T)}=0\right\}
$$

where $Q_{T}=\Omega \times(0, T)$.

Definition 2.1 A function $u \in L^{\infty}\left(Q_{T}\right)$ is called a sub-solution (super-solution) of Problem (1.1) in $Q_{T}$ if the following conditions hold:

(i) $u(x, 0) \leq(\geq) u_{0}(x)$ in $\Omega$,

(ii) $u(x, t) \leq(\geq) 0$ on $\partial \Omega \times(0, T)$,

(iii) for almost every $t \in(0, T)$ and every $\xi \in \mathcal{F}$,

$$
\begin{gathered}
\int_{\Omega} u(x, t) \xi(x, t) \mathrm{d} x-\int_{\Omega} u_{0}(x) \xi(x, 0) \mathrm{d} x+b \int_{0}^{t} \int_{\Omega} u^{r}(x, s) \xi(x, s) \mathrm{d} x \mathrm{~d} s \\
\leq(\geq) \int_{0}^{t} \int_{\Omega}\left\{u \xi_{s}+u^{m} \Delta \xi+a \int_{\Omega} u^{q}(y, s) \mathrm{d} y \xi(x, s)\right\} \mathrm{d} x \mathrm{~d} s .
\end{gathered}
$$

A function $u(x, t)$ is called a local solution of (1.1) if it is both a sub-solution and a supersolution for some $T>0$ and $u(x, t)$ is called a solution of (1.1) if it is a local solution of (1.1) in $Q_{T}$ for any $T>0$.

Local existence of weak solutions of (1.1) can be obtained by utilizing the methods of standard regularization (see [9]) and the continuity of the solutions can be derived by the arguments similar to that in [18]. Moreover, Problem (1.1) admits global solutions when the initial data are small (see [1]). Since the regularization procedure is crucial in what follows, we shall sketch the outline. Consider the regularized problem

$$
\begin{cases}u_{t}=\Delta u^{m}+a \int_{\Omega} u^{q}(y, t) \mathrm{d} y-b u^{r}, & x \in \Omega, 0<t<T, \\ u(x, t)=1 / k, & x \in \partial \Omega, 0<t<T, \\ u(x, 0)=u_{0}(x)+1 / k, & x \in \Omega,\end{cases}
$$


where $T>0$ may be chosen sufficiently small in such a way that there exists a solution $u_{k}$ of (2.2) on $Q_{T}$ for every $k \in \mathbb{N}$, and $\left\|u_{k}\right\|_{\infty}$ is bounded independently of $k$. Furthermore, $1 / l \leq u_{l} \leq u_{k}$ for $k<l$, and a super-solution (sub-solution) comparison theory holds for (2.2) (see $[1,19])$.

Since $u_{k}$ is monotone in $k$, we may define $U(x, t) \equiv \lim _{k \rightarrow \infty} u_{k}(x, t)$, and it is easy to see that $U(x, t)$ is a solution of (1.1). Furthermore, if $u$ is a solution of (1.1), then we have

$$
\begin{aligned}
\int_{\Omega}\left(u-u_{k}\right) \xi(x, t) \mathrm{d} x \\
=\int_{0}^{t} \int_{\Omega}\left\{\left(u-u_{k}\right) \xi_{s}+\left(u^{m}-u_{k}^{m}\right) \Delta \xi+a \int_{\Omega}\left(u^{q}(y, s)-u_{k}^{q}(y, s)\right) \mathrm{d} y \xi(x, s)\right\} \mathrm{d} x \mathrm{~d} s \\
\quad-b \int_{0}^{t} \int_{\Omega}\left(u^{r}(x, s)-u_{k}^{r}(x, s)\right) \xi(x, s) \mathrm{d} x \mathrm{~d} s+\frac{1}{k^{m}} \int_{0}^{t} \int_{\Omega} \frac{\partial \xi}{\partial n} \mathrm{~d} S_{x} \mathrm{~d} s-\frac{1}{k} \int_{\Omega} \xi(x, 0) \mathrm{d} x \\
\quad \leq \int_{0}^{t} \int_{\Omega}\left\{\left(u-u_{k}\right) \xi_{s}+\left(u^{m}-u_{k}^{m}\right) \Delta \xi+a \int_{\Omega}\left(u^{q}(y, s)-u_{k}^{q}(y, s)\right) \mathrm{d} y \xi(x, s)\right\} \mathrm{d} x \mathrm{~d} s,
\end{aligned}
$$

where we use the fact $\partial \xi / \partial n \leq 0$ on $\partial \Omega$ to derive this inequality. With $\Phi_{k}$ and $F_{k}$ defined so that

$$
\left(u-u_{k}\right) \Phi_{k}=u^{m}-u_{k}^{m}, \quad\left(u-u_{k}\right) R_{k}=u^{r}-u_{k}^{r}
$$

and

$$
\left(u-u_{k}\right) F_{k}=u^{q}-u_{k}^{q}
$$

we have

$$
\begin{aligned}
\int_{\Omega}\left(u-u_{k}\right) \xi(x, t) \mathrm{d} x \leq & \int_{0}^{t} \int_{\Omega}\left(u-u_{k}\right)\left\{\xi_{s}+\Phi_{k} \Delta \xi-b R_{k} \xi\right\} \mathrm{d} x \mathrm{~d} s \\
& +a \int_{0}^{t} \int_{\Omega} \xi(x, s)\left\{\int_{\Omega}\left(u-u_{k}\right) F_{k}(y, s) \mathrm{d} y\right\} \mathrm{d} x \mathrm{~d} s .
\end{aligned}
$$

Thus, we can choose the appropriate test function $\xi$ as in $[1,19]$ to obtain $u \leq u_{k}$. If $u$ is a sub-solution of (1.1), the above argument shows that $u \leq u_{k}$. Thus $U(x, t)$ is the maximal solution of (1.1), and this solution satisfies a sub-solution comparison principle.

Before proving our main results, we give a comparison principle for the solution of Problem (1.1), which is similar to Proposition 2.3 in [9] and can be proved by modifying the above arguments (see also $[1,10,19]$ ).

Proposition 2.1 Let $u$ and $v$ be a nonnegative bounded sub-solution and a nonnegative super-solution of (1.1), respectively. If either $q \geq 1$ and $u$ is bounded from the above or $0<$ $q<1$ and $v$ has a positive lower bound, then $u(x, t) \leq v(x, t)$ in $Q_{T}$ if $u_{0}(x) \leq v_{0}(x)$ in $\Omega$.

Proof of Theorem 1.2 Case (i): $q=m$ with $a \mu<1$. For any bounded smooth domain $\Omega^{\prime}$ such that $\Omega^{\prime} \supset \supset \Omega$, let $\phi(x)$ be the unique solution of the following elliptic problem:

$$
\begin{cases}-\Delta \phi(x)=1, & x \in \Omega^{\prime}, \\ \phi(x)=0, & x \in \partial \Omega^{\prime} .\end{cases}
$$


By the comparison principle for linear elliptic problem we know $\varphi(x) \leq \phi(x)$ in $\Omega$. Set $\mu_{1}=\int_{\Omega} \phi(x) \mathrm{d} x, M_{1}=\max _{x \in \bar{\Omega}^{\prime}} \phi(x)$ and $\delta=\min _{x \in \bar{\Omega}} \phi(x)$. It is well known from the strong maximum principle that $\delta>0$.

By continuity, we can choose a suitable domain $\Omega^{\prime}$ with $\Omega^{\prime} \supset \supset \Omega$ such that $a \mu_{1}<1$. Define $v(x, t)=g(t) \phi^{\frac{1}{m}}(x)$, where $g(t)$ satisfies

$$
\left\{\begin{array}{l}
g^{\prime}(t) M_{1}^{\frac{1}{m}}+\left(1-a \mu_{1}\right) g^{m}(t)=0, \quad t>0, \\
g(0)=A \geq \delta^{-\frac{1}{m}}\left\|u_{0}\right\|_{L^{\infty}(\Omega)} .
\end{array}\right.
$$

Since $0<m<1$, it follows from the theory in ODEs that $g(t)$ is nonincreasing and $g(t)=0$ for all

$$
t \geq T^{*}=\frac{M_{1}^{\frac{1}{m}}}{\left(1-a \mu_{1}\right)(1-m)} A^{1-m} .
$$

Then it can be verified that $v(x, t)$ is a super-solution of (1.1). In fact, because $q=m$ and $g^{\prime}(t) \leq 0$, we know that $v(x, t)$ satisfies the following inequalities (in the weak sense):

$$
\begin{aligned}
\frac{\partial v}{\partial t} & -\Delta v^{m}-a \int_{\Omega} v^{m}(y, t) \mathrm{d} y+b v^{r} \\
& =g^{\prime}(t) \phi^{\frac{1}{m}}(x)+g^{m}(t)-a \mu_{1} g^{m}(t)+b g^{r}(t) \phi^{\frac{r}{m}}(x) \\
& \geq g^{\prime}(t) M_{1}^{\frac{1}{m}}+\left(1-a \mu_{1}\right) g^{m}(t) \\
& =0 .
\end{aligned}
$$

In addition, $v(x, t) \geq 0$ on $\partial \Omega \times(0, T)$, for any $0<T<T^{*}$, and $v(x, 0) \geq u_{0}(x)$ by the choice of $A$. Moreover, there exists a positive constant $C_{1}$ such that $v(x, t) \geq C_{1}$ in $Q_{T}$. Therefore, by applying Proposition 2.1 to (1.1) we see that $u(x, t) \leq v(x, t)$ for $(x, t) \in Q_{T}$, which implies $u(x, T) \leq v(x, T)$. The arbitrariness of $T<T^{*}$ and $v\left(x, T^{*}\right)=0$ ensure that $u\left(x, T^{*}\right)=0$. Furthermore, let $\widetilde{u}(x, t)=u\left(x, t+T^{*}\right)$, then $\widetilde{u}(x, t)$ satisfies (1.1) with the initial condition $\widetilde{u}(x, 0)=0$. By the aforementioned proof, we see that $\widetilde{u}(x, t) \leq v(x, t)$ with any $A>0$. From the relation of the extinction time $T^{*}$ of $v(x, t)$ to $A$, it follows that $\widetilde{u}(x, t)=0$ for any $t>0$, i.e. $u(x, t)=0$ for any $t \geq T^{*}$.

Case (ii): $q>m$. Let $\phi, M_{1}$ be the same as Case (i) and denote $k_{0}=\max \left\{1, \frac{2 M_{1}^{\frac{q-m}{m}} \mu}{\int_{\Omega} \phi^{\frac{q}{m}}(x) \mathrm{d} x}\right\}$. Set $v(x, t)=k \phi^{\frac{1}{m}}(x)$ with $k=\left(\frac{1}{a k_{0} \int_{\Omega} \phi^{\frac{q}{m}}(x) \mathrm{d} x}\right)^{\frac{1}{q-m}}$, then it is easy to verify that $v(x, t)$ is a supersolution of (1.1) when $u_{0}(x)$ is sufficiently small such that $u_{0}(x) \leq k \phi^{\frac{1}{m}}(x)$ in $\Omega$. Applying Proposition 2.1 to Problem (1.1) in $Q_{T}$ for any $T>0$ we obtain $u(x, t) \leq v(x, t)$ in $Q_{T}$, which implies that $u(x, t) \leq k M_{1}^{\frac{1}{m}}$. Therefore, $u(x, t)$ satisfies

$$
u_{t}-\Delta u^{m}+b u^{r} \leq a\left(k M_{1}^{\frac{1}{m}}\right)^{q-m} \int_{\Omega} u^{m}(y, t) \mathrm{d} y, \quad x \in \Omega, t>0 .
$$

By the choice of $k$ and $k_{0}$ it is easily verified that $a\left(k M_{1}^{\frac{1}{m}}\right)^{q-m} \mu \leq \frac{1}{2}<1$. Thus, by the results of Case (i), we can conclude that the solution $u(x, t)$ vanishes in finite time when the initial data are suitably small. The proof of this theorem is complete. 
Proof of Theorem 1.3 We first prove the case $r=q<1$ with $b>a|\Omega|$. Set $v(x, t)=g(t)$ where $g(t)$ satisfies the following ordinary differential equation:

$$
\left\{\begin{array}{l}
g^{\prime}(t)+(b-a|\Omega|) g^{q}(t)=0, \quad t>0, \\
g(0)=\left\|u_{0}\right\|_{L^{\infty}(\Omega)} .
\end{array}\right.
$$

Since $0<q<1$ and $b>a|\Omega|$, we know by integrating the ODE that $g(t)$ vanishes at some finite time $T^{*}$. Moreover, as in the proof of Theorem 1.2, it can be verified that $g(t)$ is a super-solution of (1.1). Thus, by applying Proposition 2.1 to $u(x, t)$ and $g(t)$ for any $0<T<$ $T^{*}$ we know that $u(x, t)$ also vanishes at $T^{*}$.

In the case $r<\min \{q, 1\}$, let $g(t)$ satisfy the following ODE:

$$
\left\{\begin{array}{l}
g^{\prime}(t)+\left(b-a|\Omega| g^{q-r}(t)\right) g^{r}(t)=0, \quad t>0, \\
g(0)=g_{0},
\end{array}\right.
$$

where $0<g_{0}<\left(\frac{b}{a|\Omega|}\right)^{\frac{1}{q-m}}$. Similar to the first case, it is well known that $g(t)$ vanishes in finite time since $r<1$ and $g(t)$ is a super-solution of (1.1) provided that $u_{0}(x)$ is small enough such that $\left\|u_{0}\right\|_{L^{\infty}(\Omega)} \leq g_{0}$. Applying Proposition 2.1 to $u(x, t)$ and $g(t)$ guarantees the finite time extinction of $u(x, t)$. This completes the proof of Theorem 1.3.

Proof of Theorem 1.4 (i) Consider first the case $q<r \leq m$. Let $\lambda_{1}$ be the first eigenvalue of the following eigenvalue problem:

$$
\begin{cases}-\Delta \psi(x)=\lambda \psi(x), & x \in \Omega, \\ \psi(x)=0, & x \in \partial \Omega,\end{cases}
$$

and $\psi_{1}(x)>0(x \in \Omega)$ be the corresponding eigenfunction. We may normalize $\psi_{1}(x)$ such that $\left\|\psi_{1}\right\|_{L^{\infty}(\Omega)}=1$. Denote $\gamma=\int_{\Omega} \psi_{1}^{\frac{q}{m}}(x) \mathrm{d} x$ and let $g(t)$ satisfy the ODE problem

$$
\left\{\begin{array}{l}
g^{\prime}(t)=-\lambda_{1} g^{m}(t)+a \gamma g^{q}(t)-b g^{r}(t), \quad t>0, \\
g(0)=0, \\
g(t)>0, \quad t>0 .
\end{array}\right.
$$

It is easy to check that $g(t)$ is nondecreasing and bounded from above by $\min \left\{\left(\frac{a \gamma}{\lambda_{1}}\right)^{\frac{1}{m-q}}\right.$, $\left.\left(\frac{a \gamma}{b}\right)^{\frac{1}{r-q}}\right\}$. Set $v(x, t)=\rho g(t) \psi_{1}^{\frac{1}{m}}(x)$. We shall show that $v(x, t)$ is a sub-solution of $(1.1)$ when $\rho>0$ is sufficiently small. In fact, simple computations show that

$$
\frac{\partial v}{\partial t}=\rho\left(-\lambda_{1} g^{m}(t)+a \gamma g^{q}(t)-b g^{r}(t)\right) \psi_{1}^{\frac{1}{m}}(x)
$$

and

$$
\begin{aligned}
& \Delta v^{m}+a \int_{\Omega} v^{q}(y, t) \mathrm{d} y-b v^{r} \\
& \quad=-\lambda_{1} \rho^{m} g^{m} \psi_{1}+a \gamma \rho^{q} g^{q}-b \rho^{r} g^{r} \psi_{1}^{\frac{r}{m}} .
\end{aligned}
$$


For $v(x, t)$ to be a sub-solution of (1.1), it suffices to show that

$$
\lambda_{1} g^{m}(t) \rho^{m}+b g^{r}(t) \rho^{r} \leq a \gamma g^{q}(t)\left(\rho^{q}-\rho\right),
$$

which follows from

$$
C\left(\rho^{m-q}+\rho^{r-q}\right) \leq \operatorname{a\gamma }\left(1-\rho^{1-q}\right)
$$

where $C=\max _{t>0}\left\{\lambda_{1} g^{m-q}(t), b g^{r-q}(t)\right\}<+\infty$. It is easy to see that (2.10) is valid for sufficiently small $\rho>0$ since $q<r \leq m<1$.

Next, we turn our attention to construct a super-solution of (1.1). Set $w(x, t)=L$, where $L=\max \left\{\left\|u_{0}\right\|_{L^{\infty}(\Omega)},\left(\frac{a|\Omega|}{b}\right)^{\frac{1}{r-q}}, \rho \max _{t \geq 0} g(t)\right\}$. Then it is not hard to see that $w(x, t)$ is a supersolution and $v(x, t) \leq w(x, t)$. Therefore, by an iteration process, one can obtain a solution of Problem (1.1), which satisfies $v(x, t) \leq u(x, t) \leq w(x, t)$. Indeed, define $u_{1}(x, t)=w(x, t)$ and $\left\{u_{k}(x, t)\right\}_{k=2}^{\infty}$ iteratively to be a solution of the problem

$$
u_{k t}-\Delta u_{k}^{m}+b u_{k}^{r}=a \int_{\Omega} u_{k-1}^{q}(x, t) \mathrm{d} x, \quad x \in \Omega, t>0,
$$

subject to the boundary and initial conditions as that in (1.1). By applying the comparison technique used in the proof of Lemma 2.1 in $[1,12]$ we know that the function $u(x, t)=$ $\lim _{k \rightarrow \infty} u_{k}(x, t)$, for every $x \in \bar{\Omega}$ and $t>0$, is a solution of (1.1). Because $v(x, t)$ does not vanish, neither does $u(x, t)$.

(ii) The case $q<m<r$ can be treated similarly to Case (i).

(iii) Finally we consider the case $r=q<m$ with $a \gamma>b$. Let $g(t)$ satisfy the following ODE:

$$
\left\{\begin{array}{l}
g^{\prime}(t)=-\lambda_{1} g^{m}(t)+(a \gamma-b) g^{q}(t), \quad t>0, \\
g(0)=0, \\
g(t)>0, \quad t>0 .
\end{array}\right.
$$

Then $g(t)$ is nondecreasing and satisfies $g(t) \leq\left(\frac{a \gamma-b}{\lambda_{1}}\right)^{\frac{1}{m-q}}$. (The upper bound of $g(t)$ can be obtained by contradiction arguments and the monotonicity of $g(t)$ follows immediately as the upper bound is derived.) As in the proof of Case (i), we can construct a non-extinction sub-solution $v(x, t)=\rho g(t) \psi^{\frac{1}{m}}(x)$ with $\rho>0$ sufficiently small.

To construct a super-solution, consider the following eigenvalue problem:

$$
\begin{cases}-\Delta \psi(x)=\lambda \psi(x), & x \in \widetilde{\Omega}, \\ \psi(x)=0, & x \in \partial \widetilde{\Omega}\end{cases}
$$

where $\widetilde{\Omega} \supset \Omega$ is a bounded domain with smooth boundary $\partial \widetilde{\Omega}$. Let $\widetilde{\lambda}_{1}$ and $\widetilde{\psi}_{1}(x)>0(x \in$ $\widetilde{\Omega})$ be its first eigenvalue and the corresponding eigenfunction, respectively. We may normalize $\widetilde{\psi}_{1}(x)$ such that $\left\|\widetilde{\psi}_{1}\right\|_{L^{\infty}(\widetilde{\Omega})}=1$. Denote $\tilde{\gamma}=\int_{\Omega} \widetilde{\psi}_{1}^{\frac{q}{m}}(x) \mathrm{d} x$ and $\tilde{\delta}=\min _{x \in \bar{\Omega}} \widetilde{\psi}_{1}(x)>0$. Set $w(x, t)=k \widetilde{\psi}_{1}^{\frac{1}{m}}(x)$, then we shall show that $w(x, t)$ is a super-solution of (1.1) provided that $k>0$ is suitably large. Indeed, if $k=\max \left\{\left(\frac{a \widetilde{\gamma}}{\lambda_{1} \widetilde{\delta}}\right)^{\frac{1}{m-q}}, \widetilde{\delta}^{-\frac{1}{m}}\left\|u_{0}\right\|_{L^{\infty}(\Omega)}, \rho\left(\frac{a \gamma-b}{\lambda_{1}}\right)^{\frac{1}{m-q}}\right\}$, we 
know that $w(x, t) \geq 0$ on $\partial \Omega \times(0, \infty), w(x, 0) \geq u_{0}(x)$ in $\Omega$ and $w(x, t)$ satisfies the following inequalities (in the weak sense):

$$
\begin{aligned}
\frac{\partial w}{\partial t} & -\Delta w^{m}-a \int_{\Omega} w^{q}(x, t) \mathrm{d} x+b w^{q} \\
& =k^{m} \widetilde{\lambda}_{1} \widetilde{\psi}_{1}(x)-a k^{q} \widetilde{\gamma}+b k^{q} \widetilde{\psi}_{1}^{\frac{q}{m}}(x) \\
& \geq k^{q}\left(k^{m-q} \widetilde{\lambda}_{1} \widetilde{\delta}-a \tilde{\gamma}\right) \\
& \geq 0 .
\end{aligned}
$$

Moreover, $v(x, t) \leq w(x, t)$ by the choice of $k$. Therefore, by applying the monotonicity iteration process we can obtain a non-extinction solution $u(x, t)$ of $(1.1)$ satisfying $v(x, t) \leq$ $u(x, t) \leq w(x, t)$. The proof of Theorem 1.4 is complete.

Proof of Theorem 1.5 The proof of this theorem is similar to that of Theorem 1.4, so we only sketch the outline here. Set $v(x, t)=\rho g(t) \varphi^{\frac{1}{m}}(x)$ where $\varphi(x)$ is defined in (1.2) and $g(t)$ satisfies the following ODE problem:

$$
\left\{\begin{array}{l}
g^{\prime}(t)=M^{-\frac{1}{m}}\left\{(a \mu-1) g^{m}(t)-b M^{\frac{r}{m}} g^{r}(t)\right\}, \quad t>0, \\
g(0)=0, \\
g(t)>0, \quad t>0 .
\end{array}\right.
$$

Since $a \mu>1$ and $m<r$, it is well known that $g(t)$ is nondecreasing and bounded above by $\left(\frac{a \mu-1}{b M^{\frac{r}{m}}}\right) \frac{1}{r-m}$. Then $v(x, t)$ is a sub-solution of $(1.1)$ if $\rho>0$ is sufficiently small. On the other hand, the super-solution $w(x, t)$ can be chosen to be a large positive constant $L$ satisfying $L \geq \max \left\{\left\|u_{0}\right\|_{L^{\infty}(\Omega)},\left(\frac{a|\Omega|}{b}\right)^{\frac{1}{r-q}}, \rho M^{\frac{1}{m}} \max _{t \geq 0} g(t)\right\}$. It can be observed that $(v, w)$ is a pair of sub-solution and super-solution of (1.1) satisfying $v(x, t) \leq w(x, t)$. Therefore, by monotonicity iteration, we know that (1.1) admits at least one solution $u(x, t)$ such that $v(x, t) \leq u(x, t) \leq w(x, t)$. Since $v(x, t)>0$ in $\Omega \times(0,+\infty), u(x, t)$ cannot vanish at any finite time. The proof of Theorem 1.5 is complete.

Proof of Theorem 1.6 (i) Let $u(x, t)$ be any solution of (1.1). It can be verified that, for the case $m=q<r<1$, a sufficiently large constant $L$ is a super-solution of (1.1). Therefore, we know that $u(x, t) \leq L$ in $\Omega \times(0,+\infty)$. For convenience, in the following proof, we assume that the weak solution is appropriately smooth; otherwise, we can consider the corresponding regularized problem, and the same result can also be obtained through an approximate process (see [15]). Multiplying equation (1.1) by $u^{m}$ and integrating by parts over $\Omega$ yield the identity

$$
\frac{1}{m+1} \frac{\mathrm{d}}{\mathrm{d} t} \int_{\Omega} u^{m+1} \mathrm{~d} x+\int_{\Omega}\left|\nabla u^{m}\right|^{2} \mathrm{~d} x+b \int_{\Omega} u^{m+r} \mathrm{~d} x=a\left(\int_{\Omega} u^{m} \mathrm{~d} x\right)^{2}
$$

Recall the embedding theorem

$$
\int_{\Omega}\left|\nabla u^{m}\right|^{2} \mathrm{~d} x \geq \lambda_{1} \int_{\Omega} u^{2 m} \mathrm{~d} x .
$$


Combining this result with (2.13) and using Hölder's inequality on the right hand side of (2.13) one obtains

$$
\frac{1}{m+1} \frac{\mathrm{d}}{\mathrm{d} t} \int_{\Omega} u^{m+1} \mathrm{~d} x+\lambda_{1} \int_{\Omega} u^{2 m} \mathrm{~d} x+b \int_{\Omega} u^{m+r} \mathrm{~d} x \leq a|\Omega| \int_{\Omega} u^{2 m} \mathrm{~d} x .
$$

Noticing that $a|\Omega| \leq \lambda_{1}$ and $u(x, t) \leq L$, we see from (2.14) that

$$
\frac{\mathrm{d}}{\mathrm{d} t} \int_{\Omega} u^{m+1} \mathrm{~d} x+b(m+1) L^{r-1} \int_{\Omega} u^{m+1} \mathrm{~d} x \leq 0
$$

which implies

$$
\int_{\Omega} u^{m+1} \mathrm{~d} x \leq e^{-b(m+1) L^{r-1} t} \int_{\Omega} u_{0}^{m+1} \mathrm{~d} x .
$$

This shows that $\|u(\cdot, t)\|_{m+1}$ tends to 0 exponentially as $t \rightarrow+\infty$.

(ii) Let $w(x, t)=g(t) \varphi^{\frac{1}{m}}(x)$, where $g(t)$ satisfies

$$
\left\{\begin{array}{l}
g^{\prime}(t)+b M^{-\frac{1-r}{m}} g^{r}(t)=0, \quad t>0, \\
g(0)=A>0 .
\end{array}\right.
$$

Since $0<r<1, g(t)$ is nonincreasing and $g(t)=0$ for $t \geq T^{*}=\frac{A^{1-r}}{b M^{-\frac{1-r}{m}}(1-r)}$. Noticing $m=$ $q$ and $a \mu=1$, one can see that $w(x, t)$ is a super-solution of (1.1) provided that $u_{0}(x) \leq$ $A \varphi^{\frac{1}{m}}(x)$ in $\Omega$. By using the arguments similar to that of the proof of Case (i) of Theorem 1.2 we can show that any solution $u(x, t)$ of Problem (1.1) vanishes in finite time.

(iii) Finally we consider the case $q=m<1 \leq r$. First we construct a non-extinction subsolution of (1.1). Set $v(x, t)=h_{0} e^{-\alpha t} \varphi^{\frac{1}{m}}(x)$, where $h_{0}, \alpha$ are two positive constants to be determined. Noticing that $a \mu=1$, it is easily verified that when $r=1, v(x, t)$ is a sub-solution of (1.1) if $\alpha \geq b$ and if $h_{0}$ is so small such that $h_{0} \varphi^{\frac{1}{m}}(x) \leq u_{0}(x)$. When $r>1$, for $v(x, t)$ to be sub-solution of (1.1) it is reasonable to choose first $h_{0}$ so small such that $h_{0} \varphi^{\frac{1}{m}}(x) \leq u_{0}(x)$ and then $\alpha \geq b h_{0}^{r-1} M^{\frac{r-1}{m}}$. Next, since $r>q$ and $v(x, t)$ is bounded, we can choose a sufficiently large constant $L \geq v(x, t)$ to be a sup-solution of (1.1). Therefore, by monotonicity iteration, we can obtain a solution of (1.1) satisfying $v(x, t) \leq u(x, t) \leq L$. Since $v(x, t)$ does not vanish at any finite time, neither does $u(x, t)$. The proof of Theorem 1.6 is complete.

Competing interests

The authors declare that they have no competing interests.

Authors' contributions

All authors contributed equally to the manuscript and read and approved the final manuscript.

\section{Acknowledgements}

The authors would like to thank the referees for their valuable comments and suggestions which improve the original manuscript. The authors would also like to express their sincere gratitude to Professor Wenjie Gao for his enthusiastic guidance and constant encouragement. The project was supported by NSFC (11271154). 


\section{References}

1. Anderson, JR, Deng, K: Global existence for degenerate parabolic equations with a nonlocal forcing. Math. Methods Appl. Sci. 20, 1069-1087 (1997)

2. Vázquez, JL: The Porous-Medium Equation: Mathematical Theory. Oxford Mathematical Monographs. Oxford University Press, Oxford (2007)

3. Berryman, JG, Holland, CJ: Stability of the separable solution for fast diffusion. Arch. Ration. Mech. Anal. 74, 379-388 (1980)

4. Bonforte, M, Grillo, G, Vázquez, JL: Behaviour near extinction for the fast diffusion equation on bounded domains. J. Math. Pures Appl. 97, 1-38 (2012)

5. Galaktionov, VA, Peletier, LA, Vazquez, JL: Asymptotics of fast-diffusion equation with critical exponent. SIAM J. Math. Anal. 31, 1157-1174 (2000)

6. Galaktionov, VA, Vazquez, JL: Asymptotic behavior of nonlinear parabolic equations with critical exponents. A dynamical system approach. J. Funct. Anal. 100, 435-462 (1991)

7. Galaktionov, VA, Vazquez, JL: Extinction for a quasilinear heat equation with absorption I. Technique of intersection comparison. Commun. Partial Differ. Equ. 19, 1075-1106 (1994)

8. Galaktionov, VA, Vazquez, JL: Extinction for a quasilinear heat equation with absorption II. A dynamical system approach. Commun. Partial Differ. Equ. 19, 1107-1137 (1994)

9. Han, YZ, Gao, WJ: Extinction for a fast diffusion equation with a nonlinear nonlocal source. Arch. Math. 97, 353-363 (2011)

10. Han, YZ, Gao, WJ: Extinction and non-extinction for a polytropic filtration equation with a nonlocal source. Appl. Anal. 92(3), 636-650 (2013)

11. Leoni, G: A very singular solution for the porous media equation $u_{t}=\Delta u^{m}-u^{p}$ when $0<m<1$. J. Differ. Equ. 132 353-376 (1996)

12. Wang, YF, Yin, JX: Critical extinction exponents for a polytropic filtration equation with absorption and source. Math Methods Appl. Sci. (2012). doi:10.1002/mma.2708

13. $\mathrm{Xu}, \mathrm{XH}$, Fang, ZB, Yi, S: Extinction and decay estimates of solutions for a porous medium equation with nonlocal source and strong absorption. Bound. Value Probl. 2013, Article ID 24 (2013)

14. Yin, JX, Li, J, Jin, CH: Non-extinction and critical exponent for a polytropic filtration equation. Nonlinear Anal. 71 347-357 (2009)

15. Yin, JX, Jin, CH: Critical extinction and blow-up exponents for fast diffusive polytropic filtration equation with sources. Proc. Edinb. Math. Soc. 52, 419-444 (2009)

16. Zheng, P, Mu, CL: Extinction and decay estimates of solutions for a polytropic filtration equation with the nonlocal source and interior absorption. Math. Methods Appl. Sci. (2012). doi:10.1002/mma.2630

17. Liu, WJ, Wang, MX, Wu, B: Extinction and decay estimates of solutions for a class of porous medium equations. J. Inequal. Appl. 2007, Article ID 087650 (2007)

18. Sacks, PE: Continuity of solutions of a singular parabolic equation. Nonlinear Anal. 7, 387-409 (1983)

19. Anderson, JR: Local existence and uniqueness of solutions of degenerate parabolic equations. Commun. Partial Differ. Equ. 16, 105-143 (1991)

10.1186/1687-2770-2014-24

Cite this article as: Li and Han: Critical extinction exponents for a fast diffusion equation with nonlocal source and absorption. Boundary Value Problems 2014, 2014:24

\section{Submit your manuscript to a SpringerOpen ${ }^{\ominus}$ journal and benefit from:}

- Convenient online submission

- Rigorous peer review

- Immediate publication on acceptance

- Open access: articles freely available online

- High visibility within the field

- Retaining the copyright to your article 